\title{
Carbon influx from the island divertor of the Wendelstein-7AS stellarator
}

\author{
U. Wenzel, H. Thomsen, P. Grigull \\ Max-Planck-Institut für Plasmaphysik, Euratom Association, \\ D-17493 Greifswald, Germany
}

\begin{abstract}
Target plate fluxes $(\mathrm{C}, \mathrm{H})$ were measured in the island divertor experiment of W7-AS by CCD cameras equipped with interference filters. Strike point patterns depend strongly on the confinement regime. Carbon fluxes are given for attached divertor plasmas under HDH and normal confinement conditions. Our studies show that the basic picture of the radiation zones which has emerged from the investigation of tokamak divertors has to be completed for the island divertor by additional radiation zones in the $\mathrm{X}$-point regions.
\end{abstract}

Paper Number: P3-39

PSI17 keywords: island divertor, carbon impurities, line emission profiles, radiation sources

JNM keywords: P0500, P0600

PACS keywords: 52.55.Hc, 52.40.Hf

\section{Introduction}

Divertors in fusion devices are the main region for plasma-wall-interaction. The plasma impinges onto the target plates and the wall material is released either by physical or chemical sputtering. The neutral impurities are ionized and distributed by transport. At sufficiently high plasma temperatures the impurity radiation is localized near the target plates in the divertor (attached radiation or attached state). In this case, the strike point pattern of the hydrogen radiation and the impurity radiation pattern is very similar. This picture of plasma-surface interaction arose at the studies of tokamak divertors. We present here similar investigations of the impurity radiation pattern for attached plasmas in the island divertor of the W7-AS stellarator. 


\section{Experimental}

The target tiles of one bottom divertor shield in W7-AS (see Fig. 1) for the island divertor geometry) were routinely observed by three CCD cameras. Two cameras were operated at constant wavelength for the detection of hydrogen and carbon. The third camera was used for special investigations with changing interference filters. The unchanged wavelengths were the $H_{\alpha}$ line at $656 \mathrm{~nm}$ and a CII doublet line at $658 \mathrm{~nm}$. In order to separate both lines very narrow interference filters were chosen with a FWHM of $1.2 \mathrm{~nm}$. Using the $H_{\alpha}$ and the CII data, the influx of carbon and hydrogen from the target plates can be characterized.

\section{Data evaluation}

The temperature in the stellarator edge plasma is sufficiently high in most cases so that neutral species entering the plasma are rapidly ionized. For ionizing plasma conditions the particle flux $\Gamma$ is given by

$$
\Gamma=1 / 4 \pi * S / X B * I
$$

where $\mathrm{S}$ is the ionization rate, $\mathrm{X}$ is the excitation rate, $\mathrm{B}$ is the branching ratio (a constant number for each spectral line) and I is the measured intensity [1]. The application to the special spectral lines for CII and $\mathrm{H}$ mentioned above $(658 \mathrm{~nm}$ and $656 \mathrm{~nm}$, respectively) is discussed in detail in [2].

In general, the atomic physics factor $\mathrm{S} / \mathrm{XB}$ is a function of electron density and temperature. At densities as characteristic for the edge of W7-AS (up to $6.5 \cdot 10^{19} \mathrm{~m}^{-3}$ ) the density dependence can be neglected. The dependence on temperature, however, must be considered. For $H_{\alpha}$ the value of $\mathrm{S} / \mathrm{XB}$ is constant for $T_{e}$ above $8 \mathrm{eV}$. Thus, we can assume a constant value for the most cases. Corrections are necessary for lower temperatures. Another correction may be necessary when the hydrogen is predominantly released as hydrogen molecules [3]. For the CII wave-length $\mathrm{S} / \mathrm{XB}$ is an increasing function of the temperature (see Fig. 2). If the temperature cannot be estimated, e.g. by a probe measurement, the calculated carbon influx has a relatively high uncertainty. From the measurement of the doublet line follows only the ground state density of the doublet system. Calculations have shown that approximately the same amount of carbon ions is in the quartet system [2]. Thus, a factor of 2 can be applied to S/XB to consider for the quartet system but this is usually within the uncertainty due to the temperature dependence.

\section{Particle fluxes over the whole operation space}

Fig. 3 shows the maximum $H_{\alpha}$ intensity at the target plate as a function of the separatrix upstream density. Data is plotted for the standard discharge of W7-AS 
from two selected target tiles for which a comparison with the Langmuir probes is possible: the inner strike point at tile 13 (strike point A) and the outer strike point at tile 5 (strike point D, see [4] for details of the divertor geometry). For edge densities up to $6.2 * 10^{19} \mathrm{~m}^{-3}$ the intensities rise almost linearly. In this region $\mathrm{S} / \mathrm{XB}$ is constant so that the intensities can be interpreted as a particle flux. We do not observe a high-recycling regime with its non-linear increase of the particle flux (flux amplification). Between separatrix densities of 4 and $6.2 * 10^{19} \mathrm{~m}^{-3}$ the divertor plasma can detach. This state of the divertor plasma is characterized by a reduced $H_{\alpha}$ intensity and thus a reduced particle flux. In this region the $H_{\alpha}$ signal cannot always be interpreted as a particle flux. A comparison with the Langmuir probe results shows that the particle fluxes at the inner strike point of target tile 13 can be lower than those estimated with a constant S/XB (see Fig. 3). In these discharges the temperature is significantly lower than $8 \mathrm{eV}$. Consequently, the temperature dependence of $\mathrm{S} / \mathrm{XB}$ has to be considered. At the outer strike point $\mathrm{D}$ of tile 5 the temperature falls never below $8 \mathrm{eV}$ even during detachment and the $H_{\alpha}$ signal is always proportional to the particle flux over the whole operation range. Note, that there is no volume recombination in the bottom divertor which is necessary for the interpretation of the $H_{\alpha}$-signal as a particle flux.

In the attached divertor plasmas (linear relation of the particle flux to the separatrix density) we observe two different confinement regimes [5]. Below a separatrix density of $3 * 10^{19} \mathrm{~m}^{-3}$ we have the normal confinement regime (NC). Above this threshold density we observe the high-density H-Mode (HDH-mode). In the next two sections we analyze the particle flux patterns for the two regimes.

\section{Particle flux profiles in the attached state}

\subsection{Normal confinement}

Fig. 4 shows the CII and $H_{\alpha}$ profiles along target tile 5. Data is from shot 51305 $(\mathrm{t}=0.525 \mathrm{~s})$; the shot with the lowest separatrix density plotted in Fig. 3. The CII intensity was multiplied by a factor of 20 to match approximately the $H_{\alpha}$ profile. Despite the temperature dependence of S/XB for the CII transition, the correspondence of both profiles is quite good. Two strike lines were labeled with $\mathrm{B}$ and $\mathrm{D}$ according to the designation of the island fans in [4]. From field line simulations it follows that for target tiles 1-11 the island fans B and D intersect the target plates as observed. Thus, in the NC regime we observe the divertor strike points due to the island geometry and the resulting carbon influx. A rough estimation shows that the intensity difference (factor 20) is in the expected order of magnitude when assuming sputtering as the release mechanism for carbon. Assuming equal S/XB factors for both spectral lines, the inverse of the factor gives the sputtering rate which is as large as expected for physical sputtering 


\subsection{High Density H-mode}

Fig. 5 shows the CII and $H_{\alpha}$ profiles along target tile 5 in the HDH-mode. Data is from shot 50770 (scan 16 corresponding to $t=0.525 \mathrm{~s}$ ). Again, the CII intensity was matched to the $H_{\alpha}$ profile, especially the island fans $\mathrm{B}$ and $\mathrm{D}$. There are, however, two additional peaks in the carbon emission which do not have a corresponding strike point in the hydrogen emission. We conclude, that the additional CII radiation peaks do not originate from carbon released from the target plate, either dissociated from methane or physically sputtered, ionized to CII and excited. We assume that the emission is at the border between the divertor and the main plasma decoupled from the divertor plasma. In order to test this hypothesis, we use a ray-tracing calculation approach based on the calculated vacuum magnetic field for the investigated discharge [6]. The result of such modeling are the projected positions of the magnetic $\mathrm{x}$-lines onto the target. Fig. 6 shows the result for $\mathrm{x}$-line 8 together with the measured two-dimensional CII emission. Both the position and the curvature fit well to the observed radiation zone. Based on these simulations we identified the additional peaks in Fig.5 as x-lines 8 and 9 .

\section{Carbon strike point emission versus density}

Fig. 7 shows the maximum CII intensity of the strike points B and D at target tile 5. Data is given only for attached discharges. At the transition to the HDH-mode at a separatrix density of $3 * 10^{19} \mathrm{~m}^{-3}$ the CII intensity from the outer strike point $\mathrm{D}$ rises by more than a factor of two. The CII emission from the inner strike point $\mathrm{B}$ is unchanged at the transition to the HDH-mode. It follows approximately the separatrix density and, thus, the particle flux to the target tile. In contrast to the $H_{\alpha}$ emission, the CII intensity cannot directly interpreted as a carbon flux since $\mathrm{S} / \mathrm{XB}$ is dependent on temperature. Langmuir probe measurements showed a temperature drop at the transition from $\mathrm{NC}$ to $\mathrm{HDH}$. At strike point $\mathrm{D}$ on target tile 5 the temperature falls from 70 to $35 \mathrm{eV}$.

Consequently, S/XB reduces from 32 to 20 . The intensity must be corrected by the reduced factor to get the carbon flux. In Fig. 7 the CII intensities in the HDH-regime were corrected and a relative influx was plotted. Thus, the jump of the CII intensity at the $\mathrm{HDH}$ transition is only due to the temperature drop. Applying the correct value for $\mathrm{S} / \mathrm{XB}$ we find that the carbon influx from the outer strike point $\mathrm{D}$ saturates in the HDH-mode. 


\section{Summary}

Our study of attached divertor plasmas has two main results:

- In the NC regime the strike point pattern can be well understood at target tile 5. With the transition to the HDH-mode two additional carbon radiation zones appear in the lower divertor. They do not have a corresponding strike line pattern in the hydrogen emission and, consequently, they do not result from local plasma-wall-interaction. They can be well explained by carbon radiation in the $\mathrm{X}$-points. Consequently, the radiation pattern in the island divertor is composed by two different parts: The first one by plasma-wall interaction (Carbon sources at the target plates) and the second one by carbon transport (Carbon sources from the plasma volume). Note that the discharges are attached, i.e. the CII radiation from plasma-wall-interaction is attached to the target tiles.

- The carbon influx depends on the confinement regime. In the $\mathrm{NC}$ regime we observe a linear increase with the edge density while in the HDH mode the carbon flux is constant. At the HDH-transition we observe a drop of the strike point (D) temperature by a factor of two (from 70 to $35 \mathrm{eV}$ ). Flux evaluation must consider the temperature dependence of $\mathrm{S} / \mathrm{XB}$ for the special wavelength.

\section{References}

[1] P. Bogen and E. Hintz, Plasma edge diagnostics using optical methods, in NATO ASI Series B 131, 211-280 (1986).

[2] A. R. Field, C. García-Rosales, G. Lieder, C. S. Pitcher, R. Radtke, and ASDEX Upgrade Team, Spectroscopic Measurement of Target Plate Erosion in the ASDEX Upgrade Divertor, Nucl. Fusion 36(2), 119-132 (1996).

[3] S. Brezinsek, G. Sergienko, A. Pospieszczyk, P. Mertens, U. Samm, and P. Greenland, Characterization of the deuterium recycling flux in front of a graphite surface in the TEXTOR tokamak, Plasma Phys. Controlled Fusion 47, 615-634 (2005).

[4] P. Grigull, K. McCormick, Y. Feng, and A. Werner et al., Influence of magnetic field configurations on divertor plasma parameters in the W7-AS stellarator, J. Nucl. Mater. 313-316, 1287-1291 (2003).

[5] K. McCormick, P. Grigull, R. Burhenn, R. Brakel, H. Ehmler, Y. Feng, R. Fischer, F. Gadelmeier, L. Giannone, D. Hildebrandt, M. Hirsch, E. Holzhauer, R. Jaenicke, J. Kisslinger, T. Klinger, S. Klose, J. Knauer, R. König, 
G. Kühner, H. Laqua, D. Naujoks, H. Niedermeyer, E. Pasch, N. Ramasubramanian, N. Rust, F. Sardei, F. Wagner, A. Weller, U. Wenzel, and A. Werner, Island divertor experiments on the Wendelstein 7-AS stellarator, J. Nucl. Mater. 313-316, 1131-1140 (2003).

[6] H. Thomsen, R. König, Y. Feng, P. Grigull, T. Klinger, K. McCormick, N. Ramasubramanian, and $\mathrm{U}$. Wenzel, Radiative condensation and detachment in Wendelstein 7-AS stellarator, Nucl. Fusion 44, 820-826 (2004).

[7] K. Behringer, H. P. Summers, B. Denne, M. Forrest, and M. Stamp, Spectroscopic determination of impurity influx from localized surfaces, Plasma Phys. Controlled Fusion 31(14), 2059-2099 (1989). 


\section{List of Figures}

1 Vacuum magnetic configuration in the iota $=5 / 9$ configuration and the bottom divertor module (target and baffles). Letters A-D denote the intersections of the island fans with the target plate (strike points) and the numbers the nine X-points in the poloidal cross section. The CCD camera (full rectangle on top) observes the region of the bottom divertor. . . . . . . . . . . . . . . .

2 Atomic physics factor $\mathrm{S} / \mathrm{XB}$ for $\mathrm{CII}$ versus electron temperature taken from [7]. . . . . . . . . . . . . . . . . .

3 Maximum $H_{\alpha}$ emission from the divertor tiles 13 and 5 (different toroidal positions) in the bottom divertor over the separatrix density. In the attached divertor plasma the particle flux is approximately proportional to the separatrix density. . . . . . .

$4 \mathrm{CII}$ and $H_{\alpha}$ intensity profiles along target tile 5 in the normal confinement regime (discharge $51305, \mathrm{t}=0.53 \mathrm{~s}$ ). . . . . . . .

$5 \mathrm{CII}$ and $H_{\alpha}$ intensity profiles along target tile 5 in the $\mathrm{HDH}$ mode (discharge 50770, $\mathrm{t}=0.53 \mathrm{~s}$ ) . . . . . . . . . . .

6 Camera image in the light of C-II ( $\operatorname{shot} 50770$ at $\mathrm{t}=0.525 \mathrm{~s}$ ). The dashed line shows the projection of x-line 8 onto the divertor. The curvature fits well to the nearby radiation zone. The striped areas are the strike zones of island fans B and D according to field line diffusion calculation. The discussed profile in Fig. 5 is taken along the solid line. . . . . . . . . . . . . . . . .

7 Left: CII maximum intensity from the strike points over separatrix density for target tile 5. Only data from attached discharges was plotted. At the transition to the HDH-mode $\left(\right.$ at $3 * 10^{19} \mathrm{~m}^{-3}$ ) the intensity of the outer strike line rises by more than a factor of two. Right: Relative carbon influx from the outer strike point D. . . . 


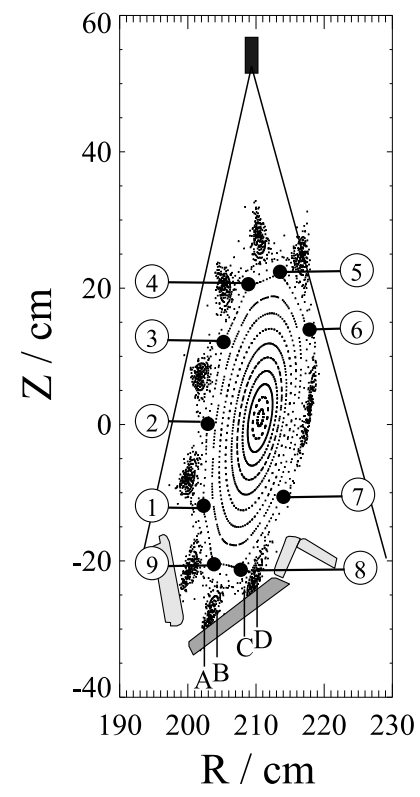

Figure 1: Vacuum magnetic configuration in the iota $=5 / 9$ configuration and the bottom divertor module (target and baffles). Letters A-D denote the intersections of the island fans with the target plate (strike points) and the numbers the nine $\mathrm{X}$-points in the poloidal cross section. The CCD camera (full rectangle on top) observes the region of the bottom divertor. 


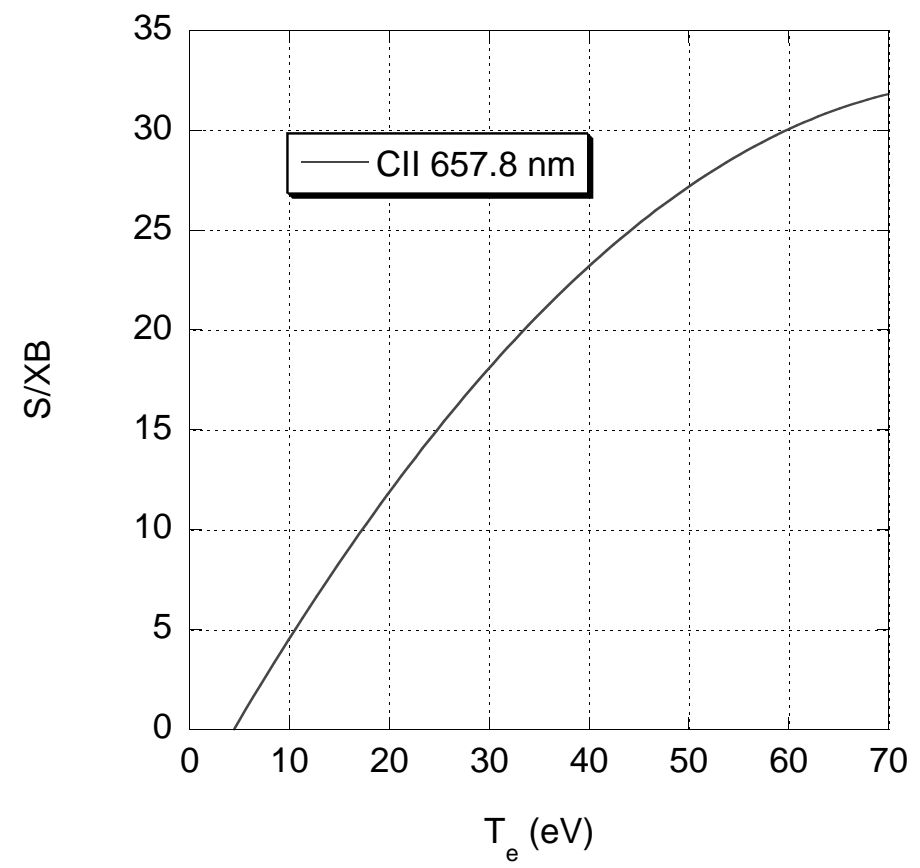

Figure 2: Atomic physics factor S/XB for CII versus electron temperature taken from [7]. 


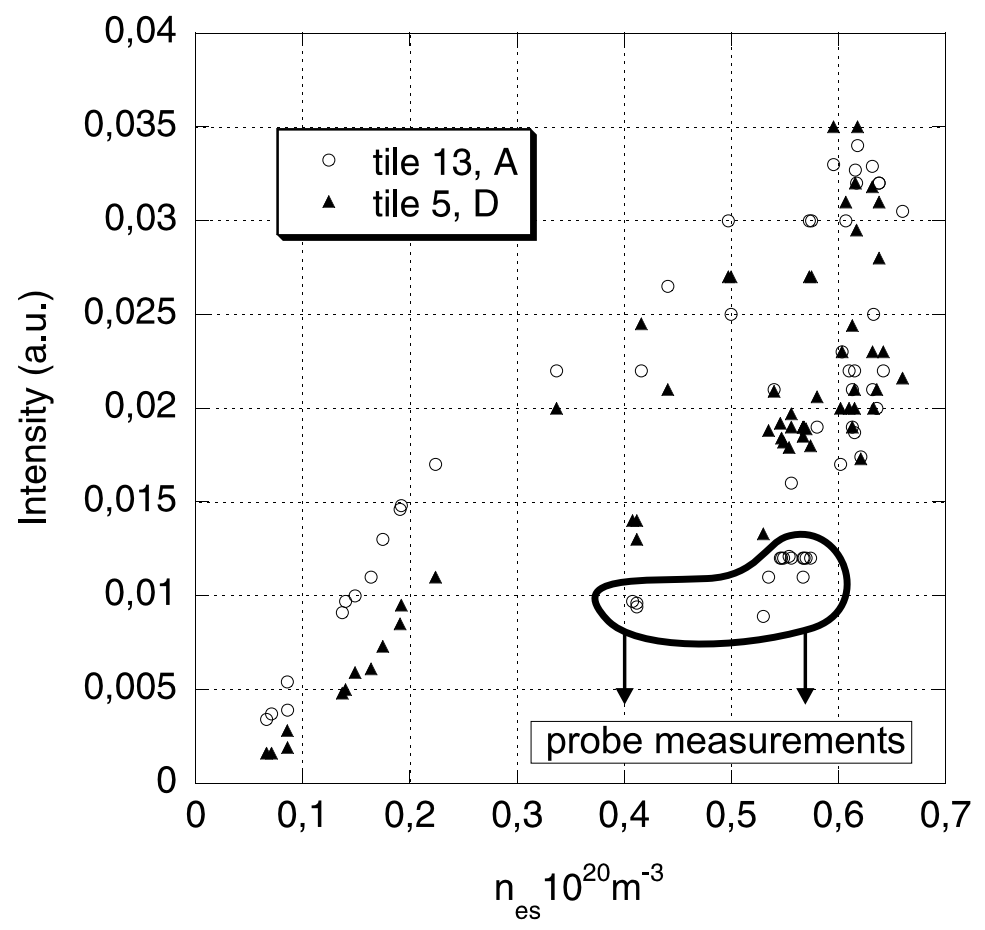

Figure 3: Maximum $H_{\alpha}$ emission from the divertor tiles 13 and 5 (different toroidal positions) in the bottom divertor over the separatrix density. In the attached divertor plasma the particle flux is approximately proportional to the separatrix density. 


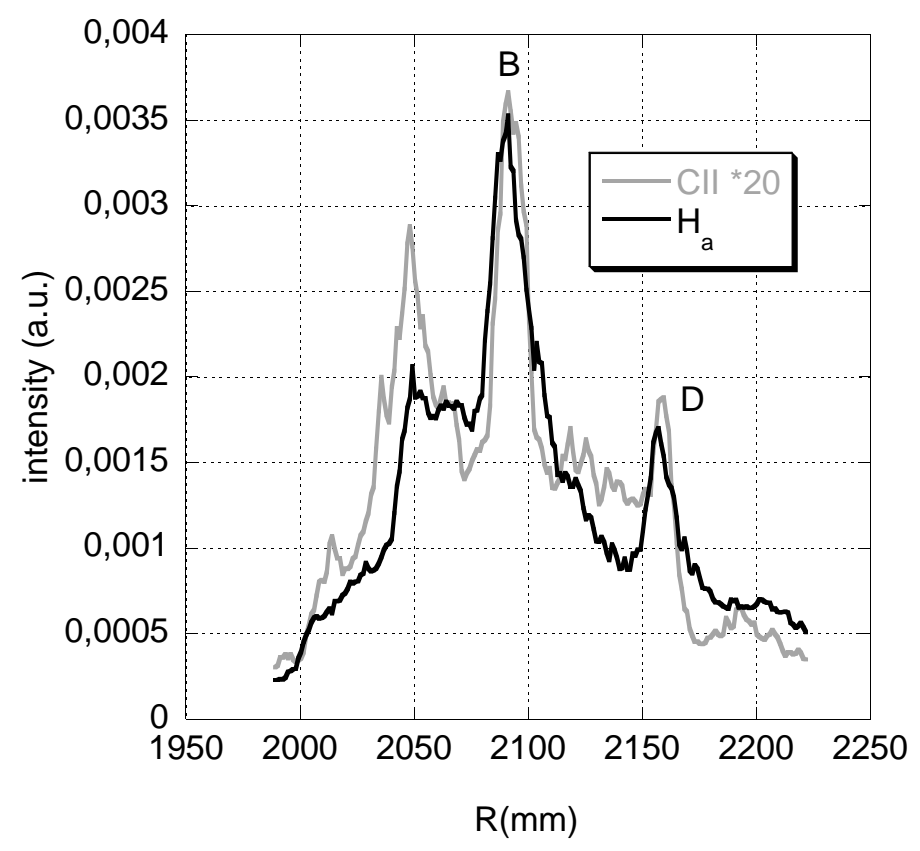

Figure 4: CII and $H_{\alpha}$ intensity profiles along target tile 5 in the normal confinement regime (discharge 51305, $\mathrm{t}=0.53 \mathrm{~s}$ ). 


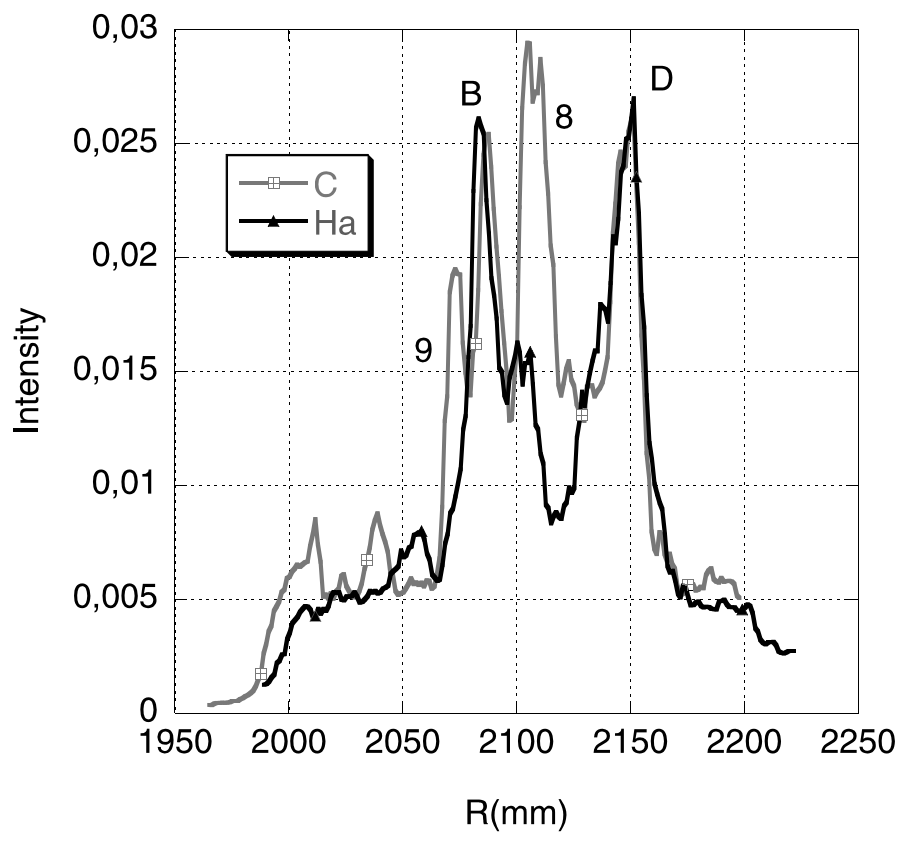

Figure 5: CII and $H_{\alpha}$ intensity profiles along target tile 5 in the $\mathrm{HDH}$ mode (discharge $50770, \mathrm{t}=0.53 \mathrm{~s}$ ). 


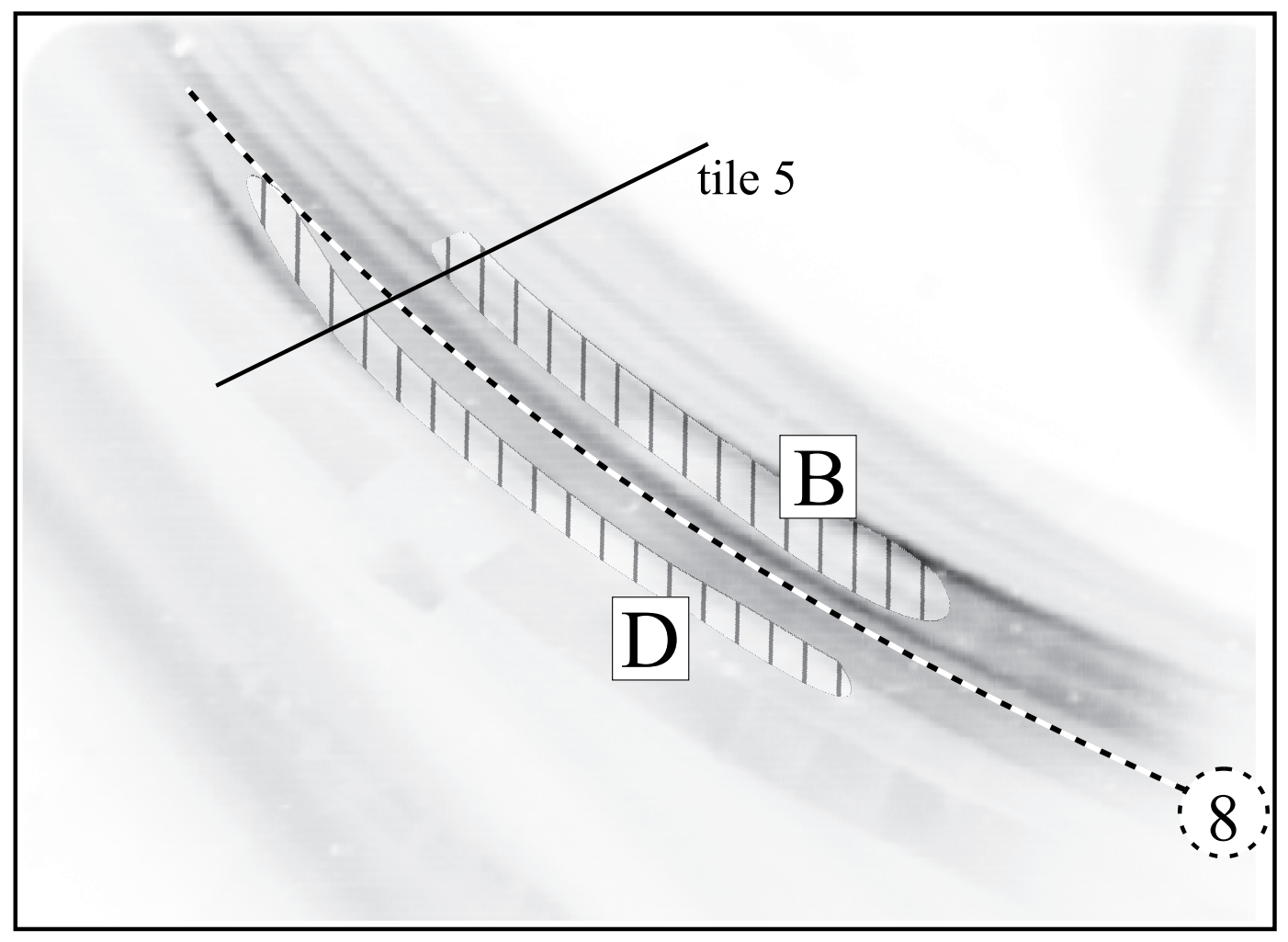

Figure 6: Camera image in the light of C-II (shot 50770 at $\mathrm{t}=0.525 \mathrm{~s}$ ). The dashed line shows the projection of $\mathrm{x}$-line 8 onto the divertor. The curvature fits well to the nearby radiation zone. The striped areas are the strike zones of island fans B and D according to field line diffusion calculation. The discussed profile in Fig. 5 is taken along the solid line. 

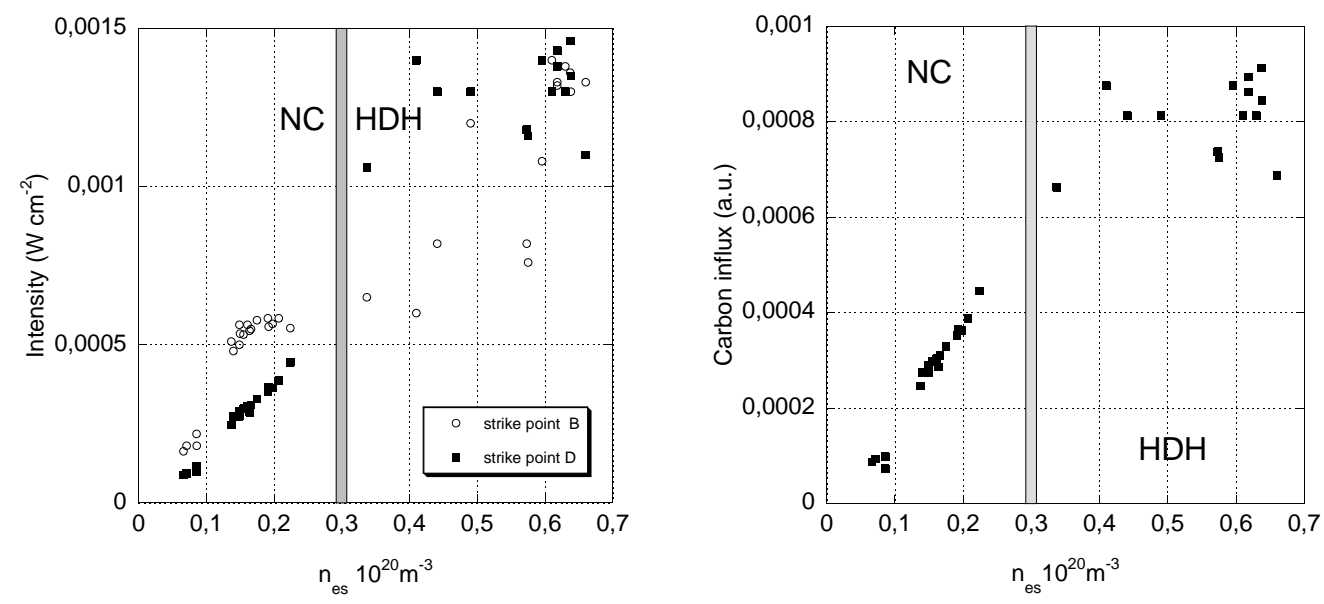

Figure 7: Left: CII maximum intensity from the strike points over separatrix density for target tile 5. Only data from attached discharges was plotted. At the transition to the HDH-mode (at $3 * 10^{19} \mathrm{~m}^{-3}$ ) the intensity of the outer strike line rises by more than a factor of two. Right: Relative carbon influx from the outer strike point D. 